\title{
Review on the Systemic Damage and Biochemical Alterations Associated with COVID-19 Infection
}

\author{
Yiglet Mebrat* \\ Correspondent to: yigletmebrat@gmail.com or yiglet.mebrat@ebi.gov.et \\ Ethiopian Biodiversity Institute, Addis Ababa, Ethiopia \\ DOI: 10.47760/cognizance.2021.v01i08.003
}

\begin{abstract}
According to the WHO daily report, the world lost 4.3 million peoples and more than 203 million of the people were infected by the epidemic till August 10, 2021. Coronavirus Disease 2019 (COVID-19) caused by severe acute respiratory syndrome coronavirus 2 (SARSCoV-2) is a respiratory disease and it is a systemic infection with cardiovascular, pulmonary, gastrointestinal, neurological, and hematological manifestations, leading to death. Many biomarkers reflecting the main pathophysiological characteristics of the disease have been associated with the risk of developing severe disease. Biochemical changes like leukocytosis, neutrophilia, lymphopenia, cytokine storm, decreased albumin, increase in ALT, total bilirubin, LDH, and procalcitonin levels are significant predictors of ICU admitted COVID-19 patients. So, monitoring of biochemical parameters in COVID-19 patients is critical for assessing disease severity and progression as well as monitoring therapeutic intervention. Keywords: SARSCoV-2, COVID-19, inflammation, biochemical alterations \& Systemic damage.
\end{abstract}

\section{Background}

The world is grappling with an unprecedented pandemic, 4.3 millions of peoples has been lost and more than 203 million of peoples has been infected (WHO report) by the proposed interim name of the virus is $2019 \mathrm{nCoV}$ (novel Corona virus, Covid-19), identified as a "severe acute respiratory syndrome coronavirus 2" (SARS-CoV-2) (Bohn et al., 2020) with incubation period of approximately 3-5 days, and some reports have put the range up to as high as 14 days (Han et al., 2020; Li et al., 2020; Verity et al., 2020). Most SARS-CoV-2-infected individuals are asymptomatic or present non-specific, flu-like symptoms, such as fever, headache, fatigue, and dry cough. However, clinical conditions can rapidly progress to severe pneumonia and, ultimately, death, mainly in patients who present co-morbidities, such as obesity, type 2 diabetes and cardiovascular diseases (Isabella et al., 2021). The disease caused by SARS-CoV-2, COVID-19, has a very wide clinical spectrum, ranging from asymptomatic cases to development 


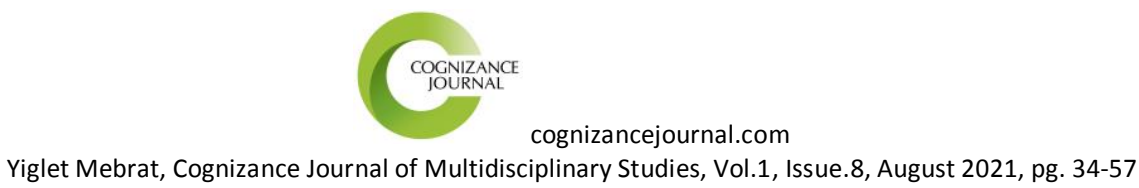

of bilateral pneumonia that can progress to respiratory failure and critically ill patients can die as the result of acute respiratory distress syndrome (ARDS) together with pneumonia in lungs and multi-organ failure (Wiersinga et al., 2020). Even the term cytokine storm has been frequently interchanged with the term 'cytokine release syndrome' (CRS), which describes an immunerelated dysregulation associated with the release of large amounts of cytokines that trigger systemic inflammation with multi-organ failure and high mortality rates (Sinha et al., 2020). The pathogenesis of immune-related muscular damage in COVID-19 is probably multifactorial, and could involve factors linked to critical illness and long ICU admissions, such as critical illness myopathy and superimposed steroid myopathy (Ramos-Casals et al., 2021). In children the disease appears to be mostly mild or asymptomatic, as a result, children, younger than a year, have an increased risk of developing a critical condition (Dong et al., 2020). People across the globe fear the impact on the health of individuals and communities, and the global economy is taking a significant hit.

This Review compiles the current knowledge of organ system (systemic) manifestations associated with the COVID-19 infection (both systemic and organ-specific) and focuses principally on specific epidemiological, biochemical, clinical and virological aspects that could help experts to identify and manage patients presenting with these features.

\section{Biochemical alteration associated with Covid-19 infection}

Monitoring of biochemical parameters in COVID-19 patients is critical for assessing disease severity and progression as well as monitoring therapeutic intervention. According to the severity of the disease, the clinical course of COVID-19 can be classified into three stages (Figure. 1), namely "early infection", "pulmonary phase", and "hyperinflammation phase", each one characterized by specific biochemical alterations (Siddiqi and Mehra, 2020). Biochemical changes (Figure 2) like leukocytosis, neutrophilia, lymphopenia, cytokine storm, decreased albumin, increase in alanine transaminase (ALT), total bilirubin, lactate dehydrogenase (LDH), and procalcitonin levels were significant predictors of ICU admitted COVID-19 patients (Jayasri et al., 2020). Biochemical findings in COVID-19 (Table 1) patients include higher levels of 
cognizancejournal.com

Yiglet Mebrat, Cognizance Journal of Multidisciplinary Studies, Vol.1, Issue.8, August 2021, pg. 34-57

AST, ALT, total bilirubin, BUN, creatinine, creatinine kinase, lactate dehydrogenase, myoglobin, creatinine kinase MB, cardiac troponin I (Henry et al., 2020).

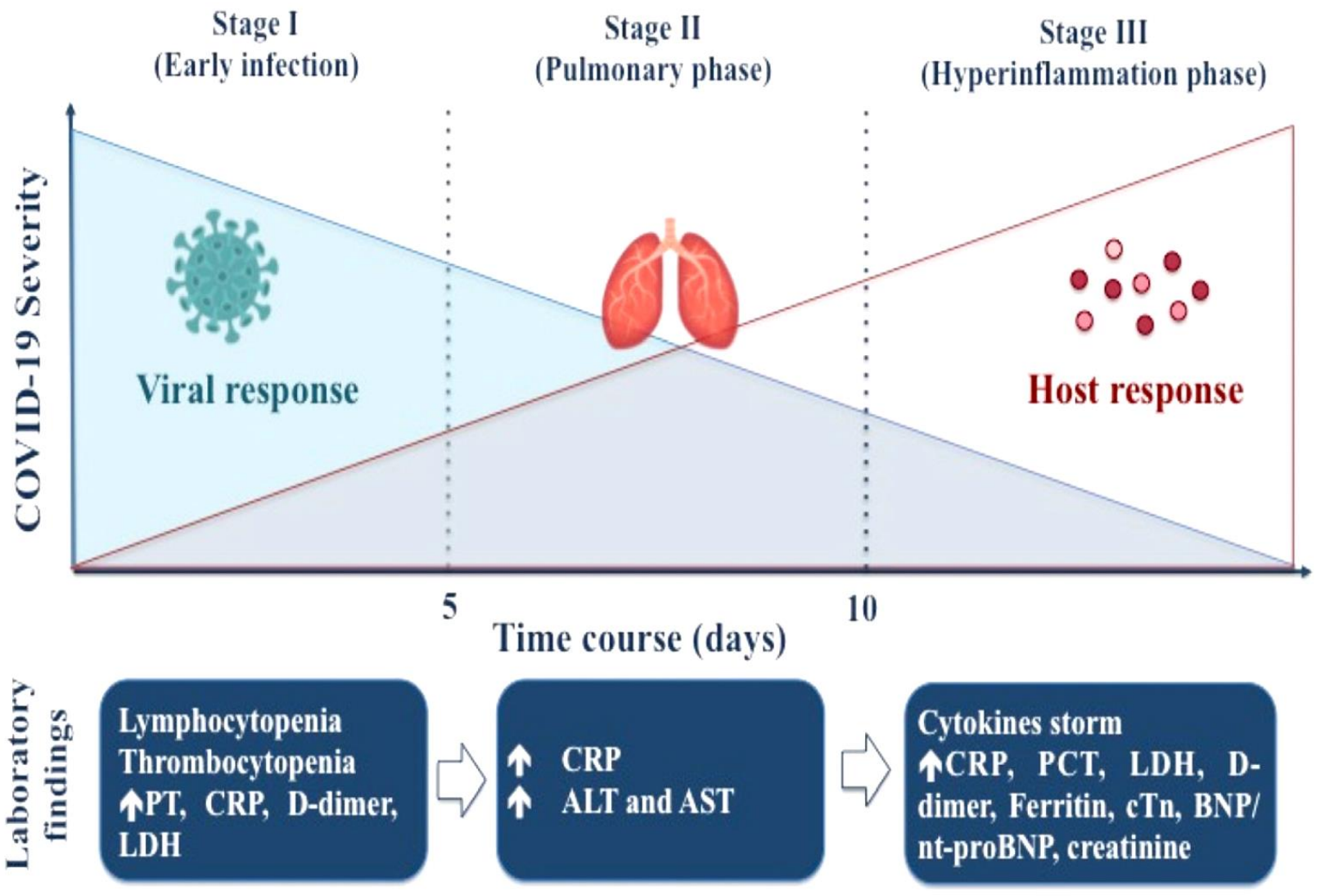

Figure 1: Disease progression and main laboratory findings (Marcello and Luisa, 2020).

Table 1: Main biochemical alterations in severe COVID-19 patients.

\section{Alteration}

Haematological

Inflammatory

Coagulation

Electrolyte

Hepatic

Muscular

\section{Biomarkers}

$\downarrow$ Lymphocyte count, $\uparrow$ Neutrophil count, $\uparrow$ NLR, $\downarrow$ Platelet count

$\uparrow \mathrm{ERS}, \uparrow \mathrm{CRP}, \uparrow \mathrm{PCT}, \uparrow$ Ferritin, $\uparrow \mathrm{IL}-6, \uparrow \mathrm{LDH}, \uparrow \mathrm{MDW}$

$\uparrow \mathrm{D}$-dimer, $\uparrow \mathrm{FDP}, \uparrow \mathrm{PT}, \uparrow$ aPTT,$\uparrow$ Fibrinogen

$\downarrow \mathrm{K}+, \downarrow \mathrm{Na}+, \downarrow \mathrm{Ca}^{++}$

$\uparrow$ Alanine aminotransferase,$\uparrow$ Aspartate aminotransferase, $\uparrow$ Total bilirubin, $\downarrow$ Albumin

$\uparrow \mathrm{CK}, \uparrow$ Mioglobin 
cognizancejournal.com

Yiglet Mebrat, Cognizance Journal of Multidisciplinary Studies, Vol.1, Issue.8, August 2021, pg. 34-57

ISSN: 0976-7797

$\begin{array}{ll}\text { Renal } & \uparrow \text { Creatinine } \\ \text { Cardiac } & \uparrow \mathrm{cTn}, \uparrow \mathrm{BNP} / \mathrm{nt}-\text { proBNP }\end{array}$

ALT, alanine aminotransferase; aPTT, activated partial thromboplastin time; AST, aspartate aminotrasnferase; BNP, brain natriuretic peptide; CK, creatine kinase; CNS, central nervous system; cTn, cardiac Troponin; CRP, c-reactive protein; FDP, fibrin degradation products; LDH, dactate dehydrogenase; NLR, neutrophils-tolymphocytes ratio; PCT, procalcitonin; PT, prothrombin time.

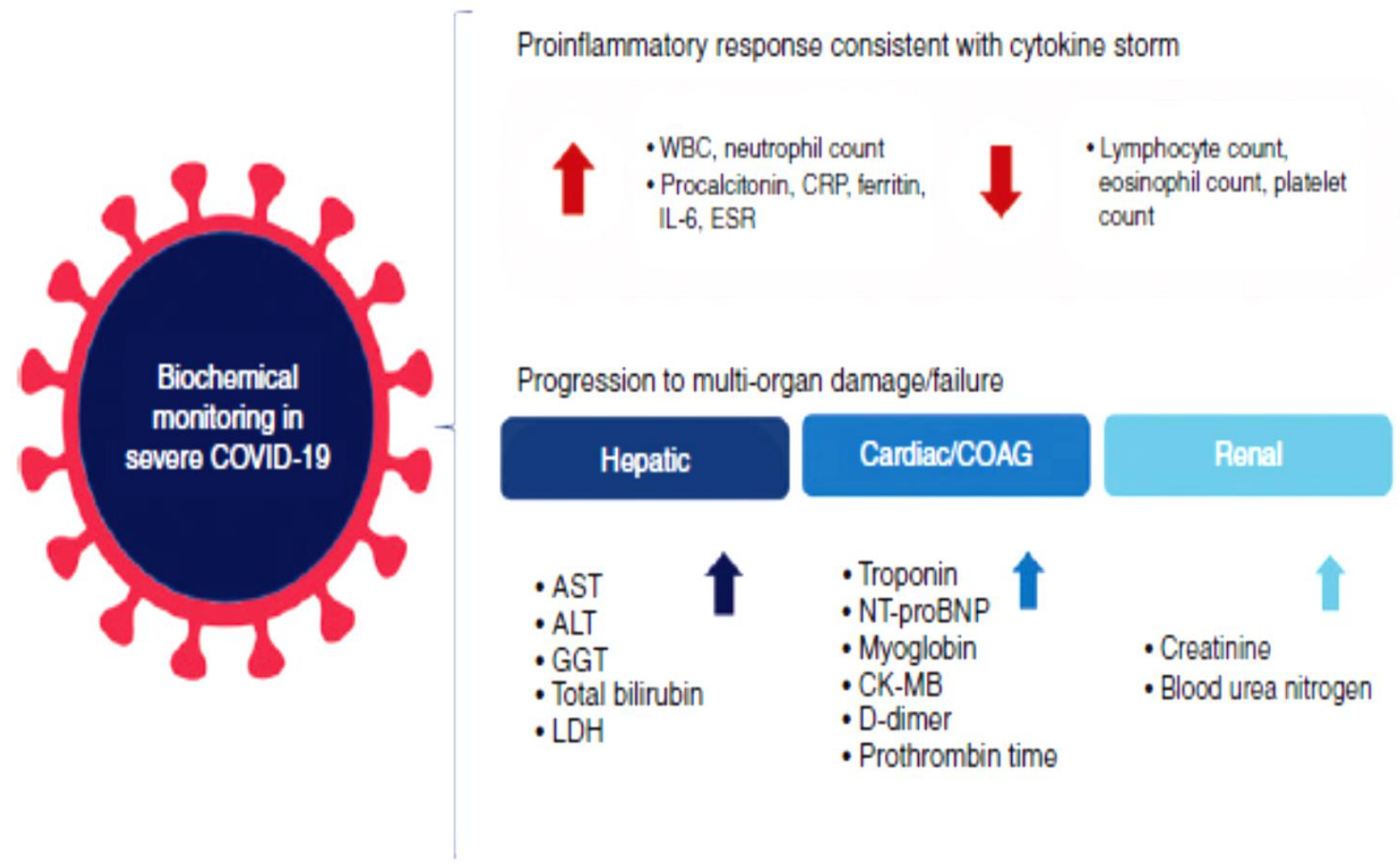

Figure 2. Biochemical and hematological biomarkers of COVID-19 progression and severity (Bohn et al., 2020).

\section{Systemic damage during Covid-19 infection}

COVID-19 is a systemic infection with cardiovascular, pulmonary, gastrointestinal, neurological, and hematological manifestations (Araya et al., 2021). Common complications observed more frequently in deceased patients included acute respiratory distress, sepsis, acute cardiac injury, heart failure, alkalosis, acute kidney injury, and hypoxic encephalopathy (Jayasri et al., 2020). Cardiovascular and hematological complications are frequent and have been associated with 


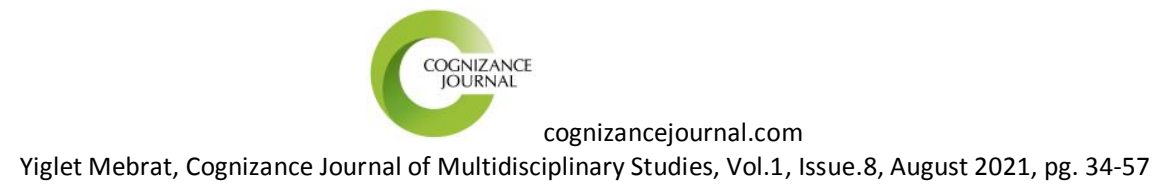

poor prognosis; gastrointestinal symptoms are also frequently encountered. Furthermore, recent studies have reported that over one-third of infected patients develop a broad spectrum of neurological symptoms. In addition, the skin, kidneys, endocrine organs, eyes and liver are also affected by systemic COVID-19 disease (Figure 3) (Fierro, 2020). Some patients with COVID19 can develop a severe, acute virus-induced lung injury under the umbrella of acute respiratory distress syndrome (ARDS), a clinical syndrome characterized by acute lung inflammation and increased-permeability pulmonary oedema due to injury to the alveolar capillary barrier (RamosCasals et al., 2021). According to proteome-wide data analysis, SARS-CoV-2 could impact several targets in diverse host tissues, making the treatment of COVID-19 patients a complex task (Mirmohammadi et al., 2020). With around 25-30\% of patients requiring intensive care due to complications like ARDS, acute lung injury, shock, and kidney failure (Hajjar and Abu-Sittah, 2021).

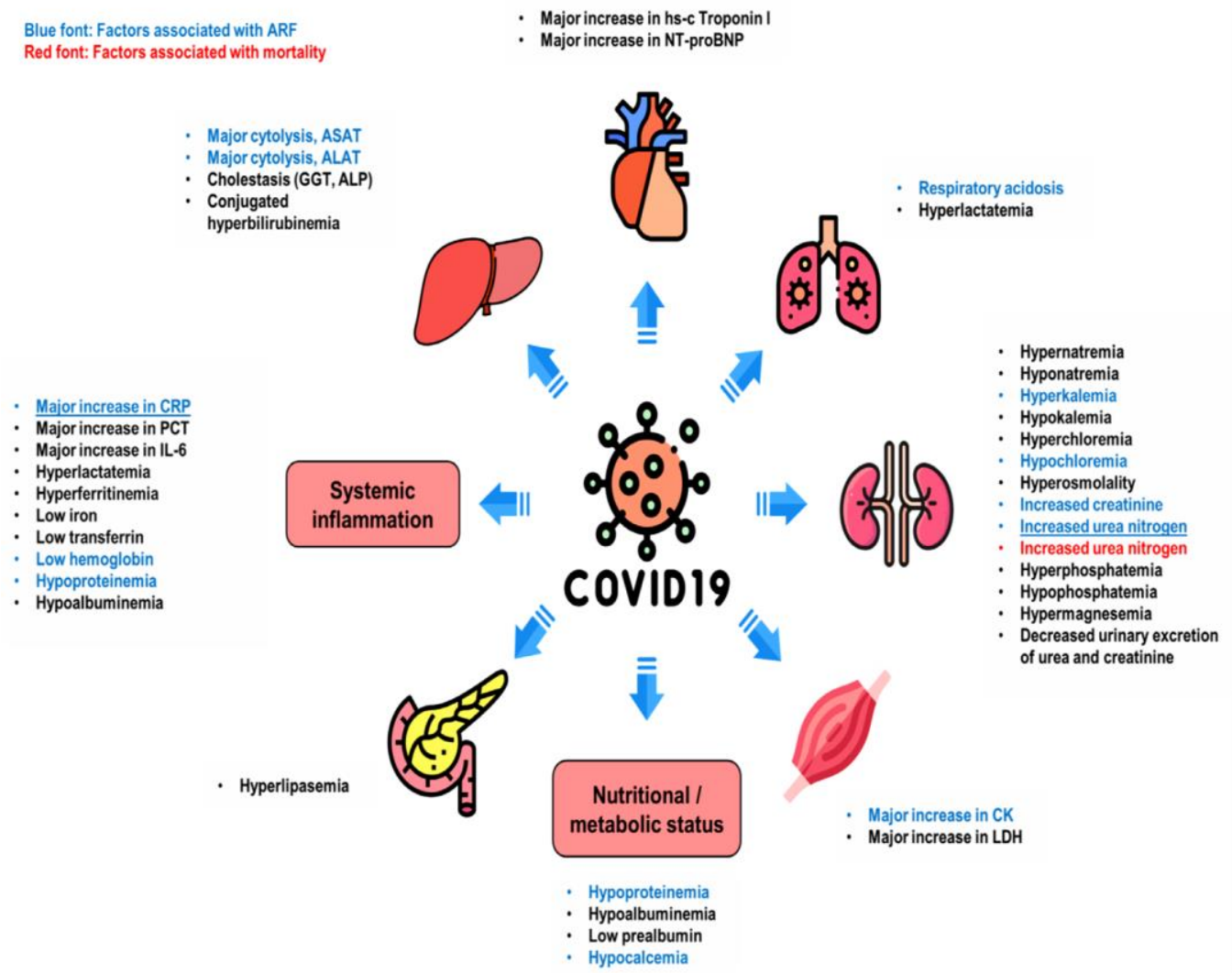

Figure 3. The major organ involvement and main biochemical abnormalities and among patients with severe COVID-19. Predictors of acute respiratory failure (ARF) in bivariate analyses are highlighted in 


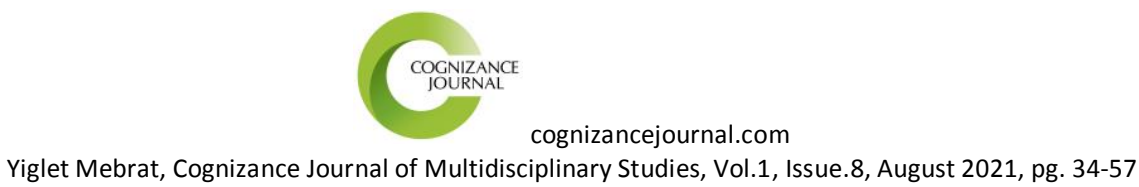

blue font. Predictors of COVID-19 related mortality in bivariate analyses are highlighted in red font. Independent predictors of ARF in the multivariable multilevel analysis are underlined. ALP: alkaline phosphatase; ASAT: aspartate aminotransferases; ALAT: alanine aminotransferases; CK: creatine kinase; CRP: C-reactive protein; GGT: g-glutamyltransferase; hs-c Troponin 1: high-sensitivity cardiac troponin I; IL-6: interleukin 6; LDH: lactate dehydrogenase; NT-pro-BNP: N-Terminal pro-Brain Natriuretic Peptide; PCT: procalcitonin; (icons made by flaticon, flaticon.com; CC-BY-3.0) (Oussalah et al., 2020).

\section{Lung injury}

The ACE2 receptor is present in the pulmonary epithelium, in type 2 pneumocytes that are present in the alveoli where the exchange between oxygen and carbon dioxide takes place, in the nose, mouth, stratified epithelial cells in the upper esophagus, and absorbent enterocytes of the ileum and colon (Troisi et al., 2021). The main target of the corona infection is the respiratory tract, and therefore, the clinical manifestations associated with the disease are mostly respiratory. Corona virus-induced acute respiratory distress syndrome (ARDS) is characterized by cytokine storm syndrome, which is a positive feedback loop that can cause a surge of pulmonary inflammation and fluid extravasation causing respiratory failure. ARDS, respiratory and/or cardiac failure, and sepsis due to secondary bacterial infection are major causes of mortality due to COVID-19 (Wei et al., 2020). According to the comparative replication capacity of SARSCoV-2 and SARS-CoV in the infected lung tissues, within the 48-hour interval, SARS-CoV-2 produced 3.2-fold more infectious virus particles than did SARS-CoV (Mirmohammadi et al., 2020).

Growing evidence supports the central role of alveolar macrophages as they secrete chemokines that induce neutrophils and polymorphonuclear cells to migrate from the bloodstream into the airspaces and alveoli. As a result of neutrophil influx and subsequent activation, these cells release toxic mediators such as reactive oxygen species, and proinflammatory mediators, such as tumour necrosis factor, interleukin-1 $\beta$ and interleukin-6, proteases and neutrophil extracellular traps, which fight invading pathogens. However, it may give rise to epithelial and endothelial injury; this weakens the alveolar-capillary barrier integrity leading to more neutrophil infiltration and surfactant dysfunction, and thereby the development of protein-rich alveolar oedema that 


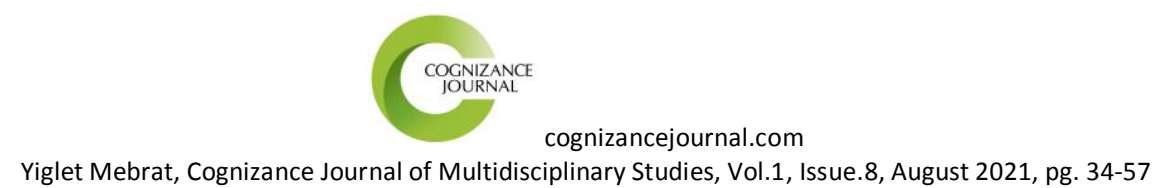

typically causes dyspnoea or difficult breathing (Zeyed et al., 2012). Importantly, the pulmonary system is by far the organ most affected by SARS-CoV-2 infection, and in severe cases the infection may develop into a serious lung injury and ARDS with eventual death (Mirmohammadi et al., 2020).

\section{Cardiac Injury associated with COVID-19 infection}

Several studies have reported the clinical and laboratory findings associated with cardiovascular disease in patients with COVID-19 infection. There is increasing evidence that a significant number of patients with COVID-19 have cardiovascular involvement, which further increases the likelihood of mortality and variety of clinical presentations of acute cardiac injury, cardiomyopathy, and hemodynamic instability (Hachim et al., 2020). Myocardial injury (Figure 4), arrhythmias, cardiac arrests, heart failure, and coagulation abnormality were reported in 7$33 \%$ of patients with COVID-19 (Huang et al., 2020). The angiotensin-converting enzyme 2 (ACE-2) receptors used for cellular entry by SARS-CoV-2 are expressed in the heart and endothelial cells (Varga et al., 2020). The cytokine storm and profound inflammation seen in patients with severe COVID-19 are associated with macrophage and endothelial activation and surges in the levels of interleukin (IL)-1, IL-6, IL-8, and Tumor Necrosis Factor-alpha (TNF-a) (Hachim et al., 2020). One of the proposed mechanisms of cardiovascular injury in COVID-19 is direct injury to myocardial cells due to viral invasion of the vascular endothelium (Figure 4) and myocardium (Clerkin et al., 2020). The second postulate is the impact of tissue hypoxia, destabilization of coronary plaque, and micro-thrombogenesis caused by the systematic inflammation associated with cytokine storm (Hachim et al., 2020). In addition, the potential role of genetic susceptibility to COVID-19 related cardiac events has recently been highlighted as a possible contributor to the high mortality among African American patients with COVID-19 (Giudicessi et al., 2020).

Cardiac injury, indicated by increased hypersensitive troponin I (hscTnI), is a common comorbidity in COVID-19 patients, reported from 12 to $77 \%$ of cases (Chen et al., 2020). Elevated hs-cTnI was also associated with death and in non-survivors, hs-cTnI increased rapidly from day 16 after disease onset, indicating that the cardiac injuries in COVID-19 might not progress linearly, but suddenly exacerbate (Chen et al., 2020; Hachim et al., 2020). Notably, even in the absence of known cardiac risk factors, patients with COVID- 19 may have an increased risk of 


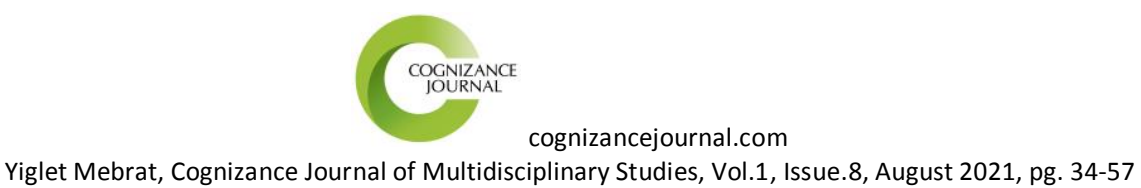

cardiovascular injury with high levels of troponin or cardiac arrest in up to $12 \%$ of patients without prior history of cardiovascular disease (Zheng et al., 2020). The value of creatine kinase isoenzyme $\mathrm{MB}$ (CK-MB) as a biomarker of myocardial injury was increased in patients with COVID-19 and was associated with severity of disease (Wang et al., 2020; Yang et al., 2020). Yang and his colleagues found that CK-MB was significantly higher in the group of patients who died among individuals with COVID-19 compared with the group of patients who survived (Mirmohammadi et al., 2020; Yang et al., 2020). Mean CK-MB was significantly higher in individuals with severe COVID-19, compared with those with less severe disease (Li et al., 2020). In addition to cTnI and CK-MB, increased lactate dehydrogenase (LDH) has been presented as a common laboratory abnormality in COVID-19 patients (Mirmohammadi et al., 2020). In a Scientific study, higher cTnI was also reported in $46 \%$ of non-survivors, in contrast to $1 \%$ of survivors (Mirmohammadi et al., 2020). Lippi et al. found that the levels of cTnI significantly increased in COVID-19 patients with severe disease compared with those without (Lippi et al., 2020).

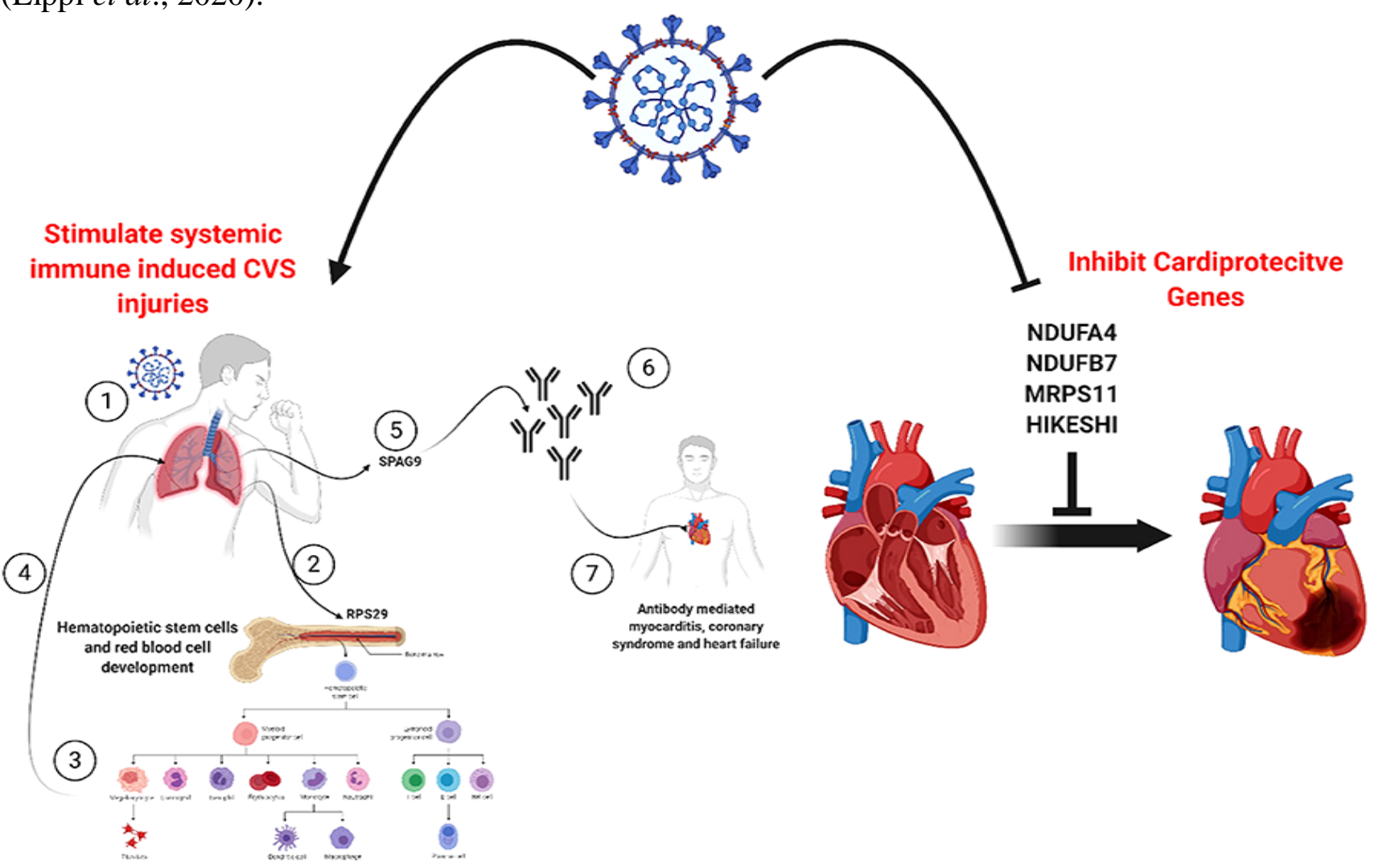

Figure 4: SARS-CoV-2 can induce cardiovascular injures in COVID-19 patients by manipulating a core set of genes specific to endothelium in the lungs, heart, and vessels. This can activate pathways for systemic immune-mediated cardiovascular injuries or increase vulnerability to cardiac injury via inhibition of cardioprotective proteins (Hachim et al., 2020). 


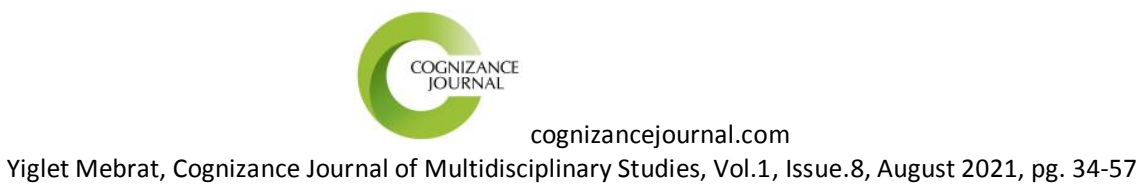

ISSN: 0976-7797

\section{Hepatic injury associated with COVID-19 infection}

COVID-19 is responsible for an acute respiratory distress syndrome. In addition to the typical respiratory signs and symptoms, mild to moderate alterations in liver function tests (LFTs) have been reported in the worst affected patients (Licata et al., 2021). Although data on COVID-19related liver abnormalities in patients remain limited, liver injury in patients is associated with prolonged hospitalization (Zhang et al., 2020). In this context, a systemic meta-analysis to assess the prevalence and degree of liver disease in severe and non- severe SARS-CoV-2 infected individuals reveals that liver injury is mostly associated with severe forms of COVID-19 rather than non-severe disease (Fierro, 2020). The extensive range of symptoms associated with COVID-19 may be related to the tropism of the virus for angiotensin converting enzyme 2 (ACE2), expressed on different human cells (Gavriatopoulou et al., 2020) and in particular, liver damage in patients with SARS-CoV-2 infection might be directly caused by the viral infection of liver cells, since ACE2 is expressed in both liver cells and bile duct cells (Prins and Olinga, 2020).

Individuals with severe COVID-19 seem to have a higher incidence of liver impairment. Most COVID-19-associated liver injuries are mild and transient, but severe liver damage can be seen (Wong et al., 2020). The incidence of liver injury can reach up to 58\% or even $78 \%$ in fatal cases and this damage may be caused by the virus itself directly infecting liver cells (Mirmohammadi et al., 2020).The mean levels of ALT, AST and bilirubin are higher in the severe COVID-19 group than in the nonsevere group (Ridruejo and Soza, 2020). This is in agreement with the elevated ALT/AST levels reported in 16-53\% of patients at the beginning of the pandemic. The liver enzyme abnormalities might also be explained by the effect of antibiotics and antiviral drugs administered to patients and inflammatory damage from the body's immune response to the virus (cytokine storm) (Fierro, 2020; Guan et al., 2020). Moreover, underlying preexisting liver diseases could contribute to liver ALT/AST elevation. In addition, the incidence of hyperbilirubinemia is 1.7-fold increased among COVID-19 patients who are critically ill, and indirect markers of liver injury, including hypoalbuminemia, have been found to be increased by seven-fold in patients with severe COVID-19 (Fierro, 2020; Xu et al., 2020). Moreover, according to the findings by Wang et al. (2020), patients who had increased transaminase levels presented higher 


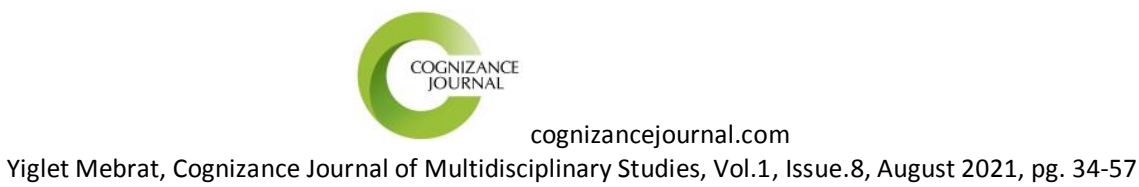

concentrations of $\gamma$-glutamyl transferase (Wang et al., 2020).It seems that liver damage is related to gender and age so that the proportion of infected patients is higher in adults than in children and in men than in women (Feng et al., 2020).

\section{Gastrointestinal tract alteration associated with COVID-19 infection}

As reported, SARS-CoV-2 infection mainly affects the respiratory system, leading to breathing difficulties, dry cough, and nasal congestion to respiratory failure, this novel coronavirus can be found in the GI tract as well (Patel et al., 2020). Tissues with the highest expression of ACE2, and therefore a high sensitivity to infection are present in both the respiratory and the gastrointestinal (GI) tracts, while ACE2-positive cells in the stomach, intestine and colon tissues, the digestive system could be susceptible to COVID-19 complications and also could act as a route of SARS-CoV-2 entry (Troisi et al., 2021). Interestingly, small intestine cells have paralleled expression of dipeptidyl peptidase-4 (a cell surface enzyme that acts as a MERS-CoV receptor) and ACE2, making them highly susceptible to beta-coronavirus infections (Mirmohammadi et al., 2020).

Several studies identified SARS-CoV-2 RNA in anal/rectal swabs (Zhang et al., 2020) and stool specimens from individuals with COVID-19 using RT-PCR (Holshue et al., 2020). The presence of SARS-CoV-2 RNA in stool samples from individuals with COVID-19 suggests potential faecal-oral transmission as an alternative infection route, so it would seem wise to take measures to minimize the spread (Mirmohammadi et al., 2020). It is noteworthy that approximately $3 \%$ of COVID-19 cases exhibited only digestive symptoms without respiratory symptoms (Ma et al., 2020). As a result, digestive symptoms are reported as the initial presentation of COVID-19 and the findings suggest that the virus can impair the digestive system and may explain the range of digestive symptoms seen in COVID-19, including diarrhea, nausea, vomiting, and diminished appetite (Ma et al., 2020). According to a cohort study of 140 hospitalized COVID-19 patients in Wuhan, gastrointestinal symptoms (39.6\%) were among the most common clinical manifestations, including nausea in $17.3 \%$, diarrhoea in $12.9 \%$ and vomiting in $5.0 \%$ (Zhang et al., 2020). So, gastrointestinal symptoms of COVID-19 can also be important for clinicians, 


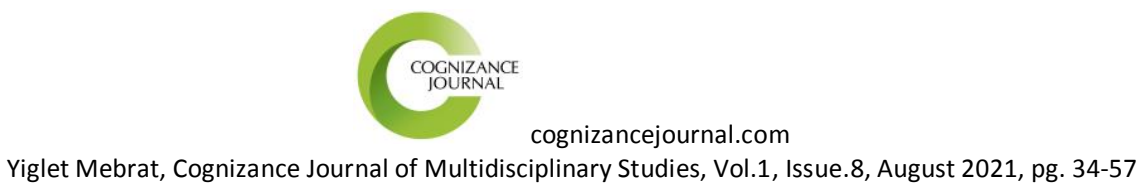

notably because they may manifest before the onset of pyrexia and respiratory symptoms (Wong et al., 2020).

Gastrointestinal endoscopy for a confirmed COVID-19 patient indicates damage to the mucosa in the esophagus, and numerous plasma cells and lymphocytes were found to have infiltrated the lamina propria of the stomach, duodenum, and rectum by histological examination. Furthermore, viral nucleocapsid protein was also detected in the cytoplasm of these sites (Ma et al., 2020). Viral nucleocapsid protein can be observed in duodenal and rectal glandular epithelial cells by laser scanning confocal microscopy. The available results provide evidence of the activity of this virus in the GI tract (Troisi et al., 2021). A report described the intestinal autopsy from a COVID-19 patient who developed alternating segmental dilatation and stenosis of the small intestine (Liu et al., 2020). Acute hemorrhagic colitis could even occur in a COVID-19 patient with digestive discomforts as the primary symptoms (Ma et al., 2020).

The pathophysiology of GI symptoms is not entirely clear, but it seems that they originate as a result of several phenomena. ACE2 performs an essential function in the intestine by regulating amino acid homeostasis and microbiome balance (Hashimoto et al., 2020). It is possible that the binding of SARS-CoV-2 to these membrane receptors reduces their availability, giving rise to an alteration of physiological function that induces a dysbiosis that leads to diarrhea, one of the most frequent symptoms reported during SARS-CoV2 infection (Troisi et al., 2021). SARSCoV-2 infection changes the amount, and blocks the ACE2 receptors in the brush edge, causing tryptophan deficiency and lower production of antimicrobial peptides, which in turn can cause changes in the intestinal microbioma and inflammation (Almeida and Chehter, 2020). Inflammation could also stimulate the gut microbiota to release molecules that increase the inflammatory state of the intestine and subsequently spread from the intestine into the circulatory system to cause systemic damage with more severe consequences than the viral infection itself (Villapol, 2020).

\section{Central nervous system}

Several respiratory viral infections, known to be neuro-tropic and neuro-invasive, such as measles virus and herpes virus, as well as influenza virus and corona-viruses are capable of 


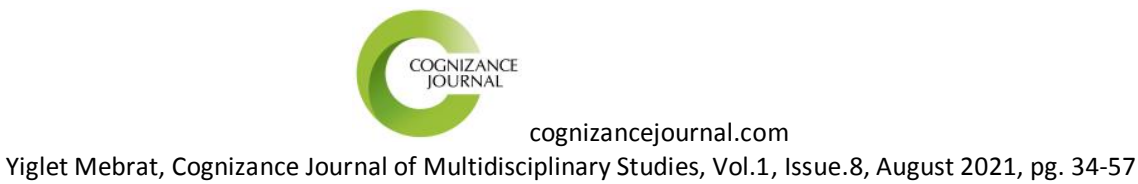

spreading throughout the body once in the lungs, and eventually infect the central nervous system (CNS) (Hosseini et al., 2021). The main target organ in SARS-CoV-2 is widely believed to be the lung; however, patients may display evidence of other organ dysfunction including the nervous system. Despite the fact that the neurotropic, neuro-invasive and neurovirulent capabilities of SARS-CoV and MERS-CoV have been described in animals and humans leading to encephalitis, meningitis or encephalomyelitis, the specific mechanisms of this process are not well characterized (Munhoz et al., 2020). In addition to lungs and various immune cells, SARS$\mathrm{CoV}$ viral particles and genomic sequences were found in neurons in the brain (Mirmohammadi et al., 2020).

Human and animal studies reports indicated that the coronavirus enters the CNS through the olfactory bulb, wherein it causes inflammation and demyelination, which is most probably behind the temporary loss of smell and/or taste in SARS-CoV-2 infection (Wu et al., 2020). Mirmohammadi et al. (2020) report on the development of non-specific neurological symptoms like dizziness, nausea, vomiting and headache as well as sudden loss of smell (anosmia) and/or taste (ageusia) by individuals with COVID-19 suggest the nervous system (Figure 5) involvement in SARS-CoV-2 infection (Mirmohammadi et al., 2020). The virus may reach and affect the CNS through several possible pathways: Direct infection injury through the blood circulation, where the vascular endothelium in the cerebral circulation express ACE2 receptors, by the neuronal pathway, by trans-synaptic transfer across infected neurons, or by an additional pathway near the olfactory bulb, through the cribriform plate (Akki et al., 2020). The brain has been reported to express ACE2 receptors that have been detected over glial cells and neurons, which makes them a potential target of COVID-19 (Mannan et al., 2020). According to genome sequencing, the presence of SARS-CoV-2 in cerebrospinal fluid has been confirmed, which may be an indication of viral encephalitis (Wu et al., 2020). Importantly, the dissemination of SARSCoV-2 to the brainstem and CNS via CSF, retrograde neuronal, and transcribrial routes can initiate an early onset of respiratory arrest by damaging medullary centers of breathing resulting in death (Mannan, 2020).

Recent reports indicate that total anosmia or partial loss of the sense of smell are early markers of SARS-CoV-2 infection, caused by "cytokine storm" initiated in some patients or direct 


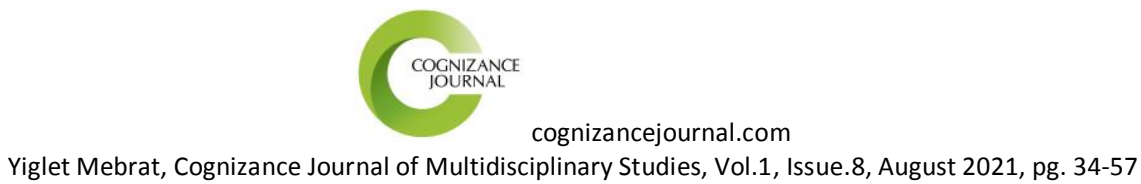

ISSN: 0976-7797

damage of the olfactory receptor neurons (ORNs) located in the olfactory epithelium and due to the fact that cells located in the olfactory epithelium (OE) express both protein receptors required for efficient SARS-CoV-2 infection in humans (Butowt and Bilinska, 2020). In the CNS, SARSCoV-2 triggers neuroinflammatory events (Figure 5), which may be a potential cause of the nervous system abnormalities initiated by ROS production and oxidative stress observed in COVID-19 patients (Akki et al., 2020). Moreover, severe hypoxia often occurs in patients with COVID-19, which may lead to subsequent nervous system damage (Wu et al., 2020).

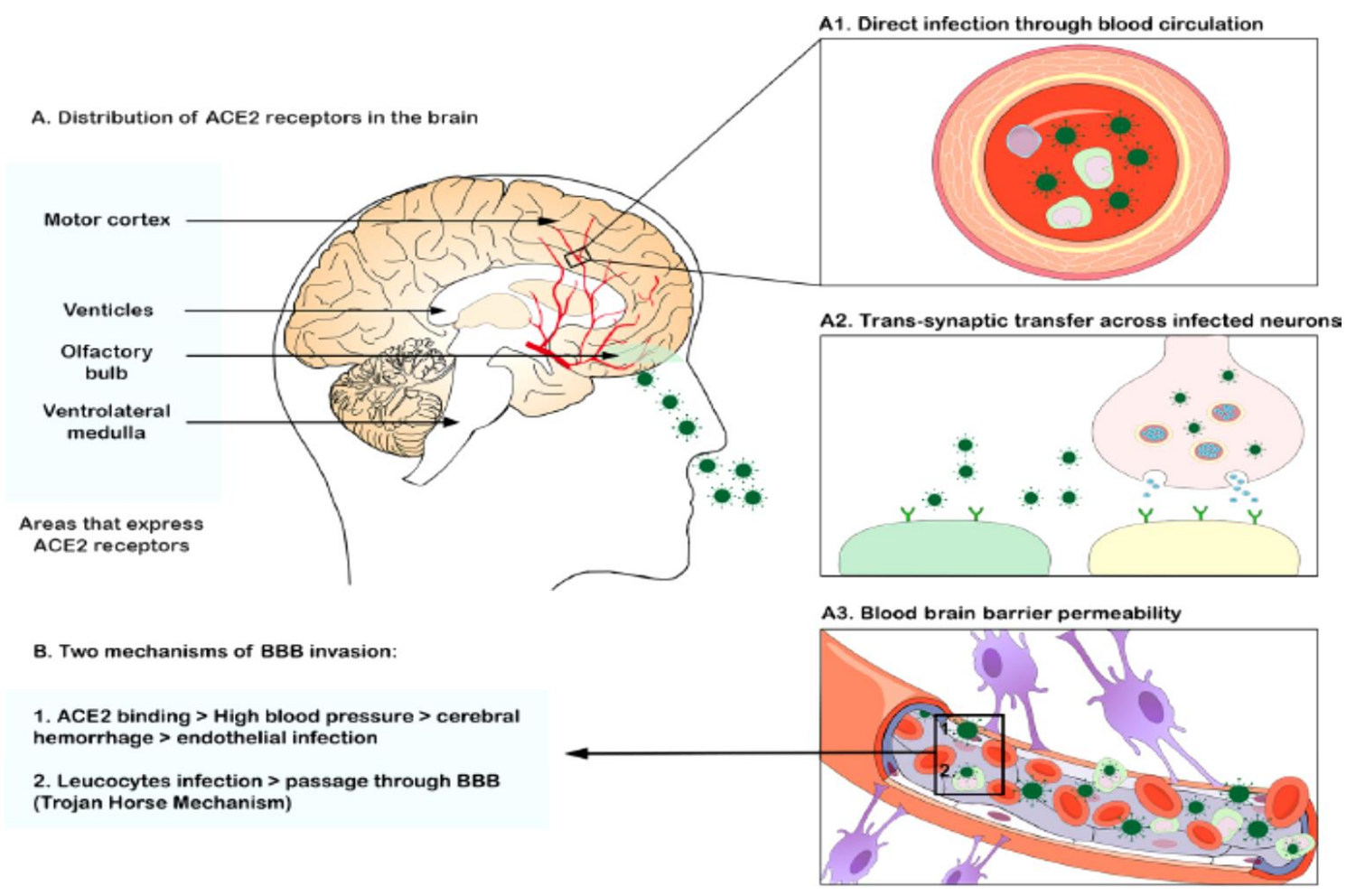

Figure 5. Distribution and expression of ACE2 in the brain and the possible pathways of SARS-CoV-2 neuroinvasion (A). The virus may enter into the brain through the blood circulation (A1), neuronal pathway (A2), olfactory bulb or via the permeable BBB (A3). It may bypass the BBB either via endothelial infection or leukocyte infection (B) (Akki et al., 2020).

\section{Haematological alterations during COVID-19 infection}

Hematological profile of severe COVID 19 patients showed increased WBC count and neutrophil count, decreased lymphocyte, platelet, eosinophil count, and hemoglobin levels (Jayasri et al., 2020). 


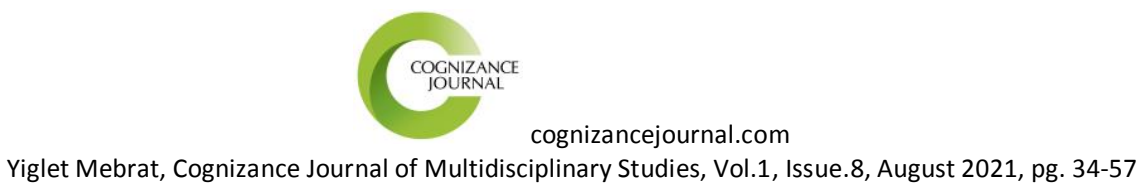

As showed for SARS-CoV, it has been hypothesized that the virus might directly infect lymphocytes, principally $\mathrm{T}$ cells, inducing depletion of $\mathrm{CD}^{+}$and $\mathrm{CD} 8^{+}$cells and, thus, suppressing the cellular immune response (Marcello and Luisa, 2020). As such in patients with severe disease, a significant increase in WBCs may signify clinical worsening and increased risk of a poor outcome. Monitoring of WBC count, lymphocyte count, platelet count, and serum ferritin can be used as markers for potential progression to critical illness particularly in hospitalized COVID-19 patients (Henry et al., 2020).

Lymphocyte percentages of COVID-19 patients indicate that the lymphocyte count is reduced with increased severity of the disease and lymphopenia is reported to be a predictor of poor prognosis in COVID-19 patients (Tan et al., 2020). A research conducted on COVID-19 patients indicated that, leukocytosis was present in 50\% patients who died and $4 \%$ who recovered and the $91 \%$ of the patients were developed lymphopenia (Jayasri et al., 2020). The mechanisms leading to lymphocyte deficiency expected to be, lymphocytes express the ACE2 receptor on their cellular membrane, the virus might directly destroy lymphatic organs and pro-inflammatory cytokines, such as IL-6 and TNF-alpha, could induce lymphocyte deficiency (Marcello and Luisa, 2020) by lymphocyte apoptosis, and inhibition of lymphocytes by metabolic molecules produced by metabolic disorders such as hyper lactic academia (Tan et al., 2020). Elevated neutrophil-to-lymphocyte ratio (NLR), resulting from the increased neutrophil count and decreased lymphocyte count, has been reported to be significantly associated with an increased risk of all-cause death during hospitalization of COVID-19 patients (Marcello and Luisa, 2020).

Elevation in the D-dimer coagulation parameter has also been consistently reported and associated with worsening disease and a higher risk of developing a wide spectrum of thromboembolic events in COVID-19 patients, including in situ pulmonary micro-thrombosis, deep vein thrombosis, overt pulmonary embolism, and even disseminated intravascular coagulation (DIC) (Bohn et al., 2020).

Thrombocytopenia has been described in COVID-19 patients and associated with the progression and prognosis of the disease (Marcello and Luisa, 2020). A meta-analysis of 1725 COVID-19 cases with 375 severe cases revealed that significantly lower platelet count in patients was associated with over fivefold enhanced risk of severity and mortality and thus, it can serve as a clinical indicator of worsening illness during hospitalization (Lippi et al., 2020). 


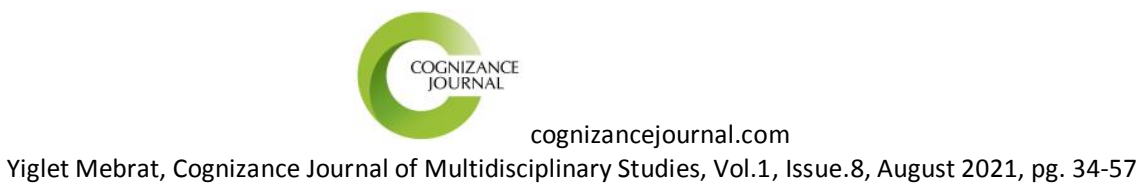

Thrombocytopenia is observed in critically ill COVID-19 patients and suggests serious organ malfunction or physiologic decompensation as opposed to primary hematologic etiology as well as the development of intravascular coagulopathy, often evolving towards disseminated intravascular coagulation. (Jayasri et al., 2020).

Several causes can induce platelet deficiency, such as the direct SARS-CoV2 infection of haematopoietic cells or bone marrow stromal cells, leading to haematopoietic inhibition (Marcello and Luisa, 2020). The lung injury could also contribute to the platelet depletion due to the activation, aggregation, and retention of platelets in the lung, and the formation of thrombus at the injured site, leading to decreased platelet production and increased consumption (Marcello and Luisa, 2020).

\section{Renal function alteration associated with COVID-19 infection}

Following lung infection with SARS-CoV-2, the virus may enter the blood circulation and, in turn, could accumulate in the kidneys resulting in renal dysfunction and its complications such as acute kidney injury (Mirmohammadi et al., 2020). In a single-cell RNA-sequencing analysis, Pan et al. concluded that the cytopathic effects of SARS-CoV-2 on podocytes and proximal straight tubule cells may lead to acute kidney injury in individuals with COVID-19, particularly in those with SARS-CoV-2 infection in blood samples (Pan et al., 2020). Furthermore, according to a meta-analysis by Henry and Lippi, chronic kidney disease appears to be associated with increased risk of severe COVID-19. The authors suggested that the presence of chronic kidney disease should be considered as an important factor in future risk stratification models for COVID-19 (Henry and Lippi, 2020).

Blood tests for assessing renal function mainly include proteinuria, urea and creatinine. A recent study also reported that among 710 hospitalized patients with COVID-19, 44\% had proteinuria, and $15.5 \%$ and $14.1 \%$ had elevated serum creatinine and blood urea nitrogen, respectively (Mirmohammadi et al., 2020). In a prospective cohort study of 701 individuals with COVID-19, it was reported that increased baseline blood urea nitrogen, increased baseline serum creatinine, proteinuria and haematuria could be independent risk factors for in-hospital death after adjusting for age, sex, disease severity, co-morbidity and leucocyte count (Cheng et al., 2020). 


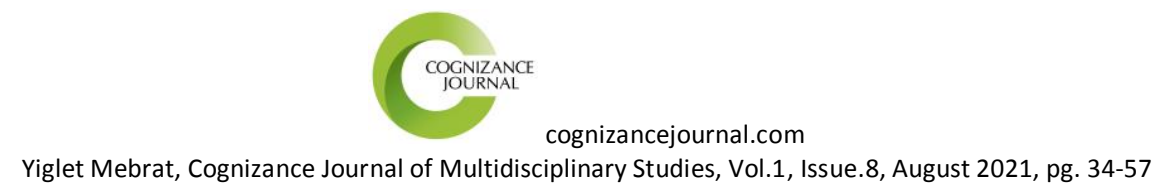

Potential mechanisms responsible for kidney injury remain to be elucidated, but sepsis and inflammation leading to cytokine storm complications and/or direct cellular injury by virus are supposed to be the case in renal impairment. Accordingly, based on electron microscope analysis in autopsies of patients with COVID-19, there were clusters of coronavirus-like particles with distinctive spikes in the tubular epithelium and podocytes ( $\mathrm{Su}$ et al., 2020).

\section{Metabolic disorder and Electrolyte imbalance}

Since the appearance of COVID-19; various reports have been published on the clinical status of infected patients and number of reports indicated hyperglycaemia and electrolyte imbalances in patients (Sarvazad et al., 2020). Hyperglycaemia can be a result of related conditions such as severe sepsis, systemic inflammatory response syndrome and traumatic brain injury. But, it was suggested that uncontrolled hyperglycaemia causes an increase in length of hospitalization and mortality caused by SARS-CoV-2 infection (Sarvazad et al., 2020). Electrolyte imbalances lead to cardiovascular and renal involvement (Post et al., 2020).

The renin-angiotensin-aldosterone system is involved in electrolyte balance and blood pressure stabilization (Muñoz-Durango et al., 2016). Electrolyte imbalance following the binding of the virus to the ACE2 receptor may contribute to metabolic disorders, in turn will lead to immune disorders, can play a role in disease prognosis and initial clinical measures and monitoring of disease (Sarvazad et al., 2020). The interaction of SARS-Cov-2 with ACE2 receptor might reduce ACE2 expression, resulting in an increased angiotensin II, which promote potassium excretion, leading to hypokalemia and lymphocytopenia (Marcello and Luisa, 2020).

Hyperglycaemia can be a result of related conditions such as severe sepsis, systemic inflammatory response syndrome and traumatic brain injury. The initial response to these conditions is an increase in the levels of some cytokines, which is accompanied by high levels of blood glucose (hyperglycaemia). It has been shown that there is a correlation between glucose blood levels and morbidity/mortality of patients (Osterbur et al., 2014). The characteristic of the pro-inflammatory phase in critical cases of these conditions is metabolic stress, which leads to the breakdown of glycogen, the synthesis of adrenocorticotrophic and glucagon hormones, and insulin resistance, which all cause an increase of blood glucose (Sarvazad et al., 2020). Wang et 


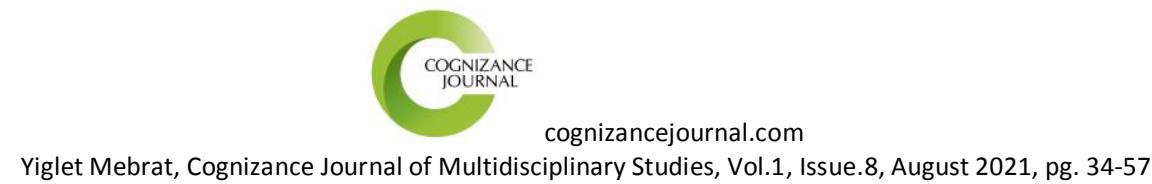

al. indicated that the level of fasting blood sugar (FBS) at the time of admission is a significant prognostic factor for COVID-19 (Wang et al., 2020). Sarvazad et al. also indicated a significant difference between the increase in the level of FBS at the time of admission between the severe and less severe COVID-19 groups (Sarvazad et al., 2020) and, consistent with the results of Wang et al., the level of FBS was higher in the ICU group than in the outpatients. However, it was suggested that acute insulin resistance, characterized by hyperglycaemia and hyperinsulinaemia, causes a higher level of glucose, which has been shown in ICU patients (BarOr et al., 2019).

Hyponatraemia has been reported in COVID-19 (Guan et al., 2019; Huang et al., 2020; Sever et al., 2020). In a retrospective study conducted by Zhang et al., the correlation between hyponatraemia and the severity of COVID-19 was considered (Zhang et al., 2020). In a study of electrolyte imbalances in patients with COVID-19, indicating a relationship between decreased blood sodium and disease severity (Lippi et al., 2020). As one of the expression sites of the ACE2 receptor is in the proximal tubule, hyponatraemia can occur due to increased expression of the ACE2 receptor in the proximal tubule. In an individual with severe hyponatraemia, it was shown that SARS-CoV-2 causes a syndrome of inappropriate secretion of antidiuretic hormone and manifestations of hyponatremia (Zhang et al., 2020).

Magnesium is known as a calcium-channel blocker and inhibits the influx of calcium to immunocompetent cells, thereby preventing the activation of nuclear factor- $\kappa \mathrm{B}$, the production of cytokines, especially interleukin-6, and also systemic inflammation (Sugimoto et al., 2012). So; the decrease in magnesium due to COVID-19 will exacerbate the inflammation caused by the virus and cause a cytokine storm. Individuals with COVID-19 manifest with hypomagnesaemia; due to stress caused by COVID-19, under the effect of stress hormones catecholamines and corticosteroids, magnesium shifts to the extracellular space and urinary excretion of $\mathrm{Mg}^{2+}$ increases (Iotti et al., 2020). As hypomagnesaemia affects the performance of the sodiumpotassium ATPase pump, as a result the prevalence of hypomagnesaemia was higher than that of hypokalaemia; this may be the reason why hypomagnesaemia precedes hypokalaemia (Sarvazad et al., 2020). Increased distal sodium delivery and aldosterone may also contribute to potassium loss along with magnesium depletion (Huang and Kuo, 2007). 


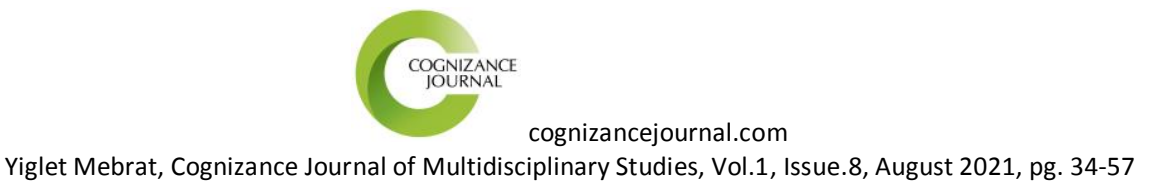

ISSN: 0976-7797

\section{Conclusion}

SARS-CoV-2 could impact several targets in diverse host tissues, making the treatment of COVID-19 patients a complex task. The presence of ACE2 receptor in many organ system and a crosstalk between organ system helps for the wide systemic alteration of COVID-19 infection and biochemical alteration. Even though the respiratory system is the main target, the central nervous system, the Gastro intestinal system, the hepatic system, the cardiovascular system, the urinary system, hematological and lymphatic systems are the target for COVID-19 alteration in consequence with death.

Overall, the scientific evidence confirms that severe cases of COVID-19 are characterized by a massive proinflammatory response or cytokine storm that is estimated to progress to multiple organ damage and failure (i.e. MOF) in severe cases. Biochemical monitoring of COVID-19 patients will thus involve assessing the inflammatory profile, as well as early recognition of cardiac, renal, and hepatic injury through routine laboratory testing to minimize the complex and systemic alteration associated with COVID-19 infection.

\section{Declarations}

\section{Ethical Approval and Consent to participate}

Since it is a review paper and no study subjects has been participating in this work, no need of ethical approval and consent to participant.

\section{Consent for publication}

I am agreed with the condition and the legal issues of the publisher since I am the correspondent author.

\section{Availability of data and materials}

No methodologies were applied to this review paper except the reported works and data in previous works were incorporated as supporting materials for the review.

\section{Competing interests}

There is no any computing interest in this review work yet. 


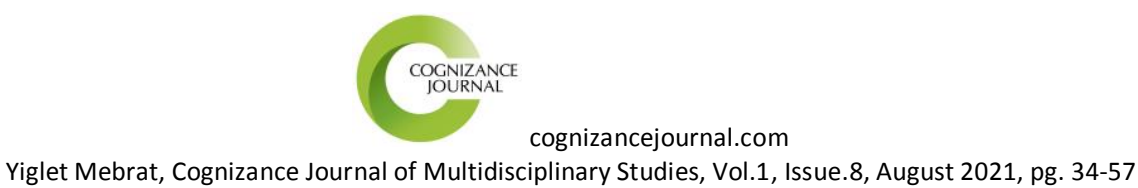

ISSN: 0976-7797

\section{Funding}

No funding to complete this work.

\section{Authors' contributions}

Except the corresponding author, no one contributes for the completion of this work.

\section{Acknowledgements}

I acknowledge my institute, Ethiopian Biodiversity Institute.

\section{Authors' information}

Yiglet Mebrat Get, Medical Biochemist, Researcher at Ethiopian Biodiversity Institute.

E-mail: yigletmebrat@gmail.com or yiglet.mebrat@ebi.gov.et

Phone: +251930834634

P.O.Box: 30726

Addis Ababa, Ethiopia

\section{References}

[1]. Almeida Joana Ferro Machado de, Chehter Ethel Zimberg. COVID-19 and the gastrointestinal tract: what do we already know? einstein (São Paulo). 2020; 18:1-14.

[2]. Akki Rachid, Nada Fath, and Hicham Mohti. COVID-19: Oxidative Preconditioning as a Potential Therapeutic Approach. ACS Chem. Neurosci. 2020; 11: 3732-3740.

[3]. Araya Shambel, Moges Wordofa, Mintesnot Aragaw Mamo, Yakob Gebregziabher Tsegay, Abebe Hordofa, Abebe Edao Negesso, Tewodros Fasil, Betelhem Berhanu, Hermela Begashaw, Asegdew Atlaw, Tirhas Niguse, Mahlet Cheru, Zemenu Tami. The Magnitude of Hematological Abnormalities among COVID-19 Patients in Addis Ababa, Ethiopia. Journal of Multidisciplinary Healthcare. 2021; 14: 545-554.

[4]. Bar-Or D, Rael LT, Madayag RM, Banton KL, Tanner A, Acuna DL, Lieser MJ, Marshall GT, Mains CW and Brody E. Stress hyperglycemia in critically ill patients: insight into possible molecular pathways. Front Med 2019; 6:54.

[5]. Bohn Mary Kathryn, Alexandra Hall, Lusia Sepiashvili, Benjamin Jung, Shannon Steele, and Khosrow Adeli. Pathophysiology of COVID-19: Mechanisms Underlying Disease Severity and Progression. PHYSIOLOGY. 2020; 35: 288-301.

[6]. Butowt Rafal and Bilinska Katarzyna. SARS-CoV-2: Olfaction, Brain Infection, and the Urgent Need for Clinical Samples Allowing Earlier Virus Detection. ACS Chem. Neurosci. 2020; 11: 1200-1203.

[7]. Chen G, Wu D, Guo W, Cao Y, Huang D, Wang H, Tao Wang, Xiaoyun Zhang, Huilong Chen, Haijing Yu, Xiaoping Zhang, Minxia Zhang, Shiji Wu, Jianxin Song, Tao Chen, Meifang Han, Shusheng Li, Xiaoping Luo. Clinical and immunological features of severe and moderate coronavirus disease 2019. J Clin Invest. 2020; 130:2620-9. 


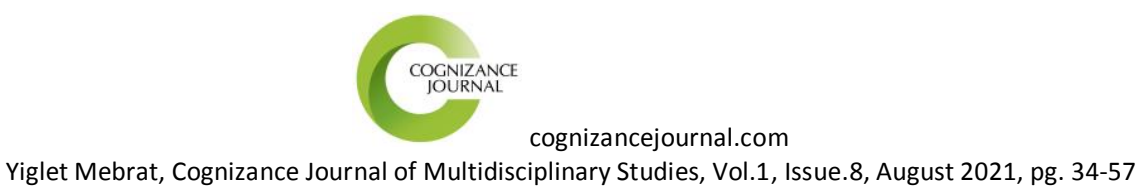

[8]. Cheng Y, Luo R, Wang K, Zhang M, Wang Z, Dong L, Junhua Li, Ying Yao, Shuwang Ge and Gang $\mathrm{Xu}$. Kidney disease is associated with in-hospital death of patients with COVID-19. Kidney Int 2020; 97:829-38.

[9]. Clerkin KJ, Fried JA, Raikhelkar J, Sayer G, Griffin JM, Masoumi A, Sneha S. Jain, Daniel Burkhoff, Deepa Kumaraiah, LeRoy Rabbani, Allan Schwartz, Nir Uriel. COVID-19 and cardiovascular disease. Circulation. 2020; 141:1648-55.

[10].Dong Yuanyuan, Xi Mo, Yabin Hu, Xin Qi, Fan Jiang, Zhongyi Jiang, Shilu Tong. Epidemiology of COVID-19 among Children in China. Pediatrics. 2020; 145(6):1-10.

[11].Feng Gong, Kenneth I. Zheng, Qin-Qin Yan, Rafael S. Rios, Giovanni Targher, Christopher D. Byrne, Sven Van Poucke, Wen-Yue Liu and Ming-Hua Zheng.. COVID19 and liver dysfunction: current insights and emergent therapeutic strategies. J Clin Transl Hepatol 2020; 8:18-24.

[12].Fierro Nora A. COVID-19 and the liver: What do we know after six months of the pandemic? Annals of Hepatology 19. 2020; 19:595-596.

[13].Gavriatopoulou M, Korompoki E, Fotiou D, Ntanasis-Stathopoulos I, Psaltopoulou T, Kastritis E, Evangelos Terpos, Meletios A. Dimopoulos. Organ-specific manifestations of COVID-19 infection. Clin Exp Med. 2020; 27:1-14.

[14].Giudicessi JR, Roden DM, Wilde AAM, Ackerman MJ. Genetic susceptibility for COVID-19-associated sudden cardiac death in African Americans. Heart rhythm. 2020; $17: 1487-92$.

[15].Guan W-J, Ni Z-Y, Hu Y, Liang W-H, Ou C-Q, He J-X, et al. Clinical characteristics of coronavirus disease 2019 in China. N Engl J Med 2020; 382(18): 1708-1720.

[16].Hachim Mahmood Yaseen, Saba Al Heialy, Abiola Senok, Qutayba Hamid and Alawi Alsheikh-Ali. Molecular basis of cardiac and vascular injuries associated with COVID19.Frontiers in Cardiovascular Medicine. 2020; 7(582399): 1-15.

[17].Hajjar MS and Abu-Sittah GS. The multidimensional burden of COVID-19 on Syrian refugees in Lebanon. J Glob Health. 2021; 11: 1-8.

[18].Han MS, Byun J-H, Cho Y, Rim JH. RT-PCR for SARS-CoV-2: quantitative versus qualitative. Lancet Infect Dis 2021; 21: 165.

[19].Henry BM, de Oliveira MHS, Benoit S, Plebani M, Lippi G. Hematologic, biochemical and immune biomarker abnormalities associated with severe illness and mortality in coronavirus disease 2019 (COVID-19): a meta-analysis. Clin Chem Lab Med. 2020; 58(7):1021-8.

[20].Henry BM and Lippi G. Chronic kidney disease is associated with severe coronavirus disease 2019 (COVID-19) infection. Int Urol Nephrol. 2020; 52:1193-4.

[21].Hashimoto T, Perlot T, Rehman A, Trichereau J, Ishiguro H, Paolino M, Sigl V, Hanada T, Hanada R, Lipinski S, Wild B, Camargo SM, Singer D, Richter A, Kuba K, Fukamizu A, Schreiber S, Clevers H, Verrey F, Rosenstiel P, Penninger JM. ACE2 links amino acid malnutrition to microbial ecology and intestinal inflammation. Nature. 2012; 487: 477481.

[22].Holshue ML, DeBolt C, Lindquist S, Lofy KH, Wiesman J, Bruce H, et al. First case of 2019 novel coronavirus in the United States. N Engl J Med 2020; 382:929-36. 


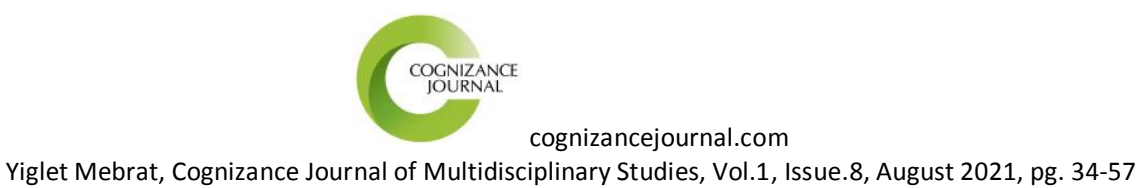

[23].Hosseini Nasrin, Nadjafi Shabnam and Ashtary Behnaz. Overview of COVID-19 and neurological complications. Rev. Neurosci. 2021; 32(6): 671-691.

[24].Huang C, Wang Y, Li X, Ren L, Zhao J, Hu Y, Zhang L, Fan G, Xu J, Gu X, Cheng Z, Yu T, Xia J, Wei Y, Wu W, Xie X, Yin W, Li H, Liu M, Xiao Y, Gao H, Guo L, Xie J, Wang G, Jiang R, Gao Z, Jin Q, Wang J, Cao B. Clinical features of patients infected with 2019 novel coronavirus in Wuhan, China. Lancet. 2020; 395:497-506.

[25].Huang C-L and Kuo E. Mechanism of hypokalemia in magnesium deficiency. J Am Soc Nephrol. 2007; 18:2649-52.

[26].Iotti S, Wolf F, Mazur A, Maier JA. The COVID-19 pandemic: is there a role for magnesium? Hypotheses and perspectives. Magnes Res 2020; 33:21-7.

[27].Isabella A. T. M. Ferreira, Dami A. Collier, Prasanti Kotagiri, Rawlings P. Datir, Eleanor Y. Lim, Emma Touizer, Bo Meng, Adam Abdullahi, The CITIID-NIHR BioResource COVID-19 Collaboration, Anne Elmer, Nathalie Kingston, Barbara Graves, Emma Le Gresley, Daniela Caputo, Laura Bergamaschi, Kenneth G. C. Smith, John R. Bradley, Lourdes Ceron-Gutierrez, Paulina Cortes-Acevedo, Gabriela Barcenas-Morales, Michelle A. Linterman, Laura E. McCoy, Chris Davis, Emma Thomson, Paul A. Lyons, Eoin McKinney, Rainer Doffinger, Mark Wills\& Ravindra K. Gupta. Age-related immune response heterogeneity to SARS-CoV-2 vaccine BNT162b2. Nature. 2021; 1-23.

[28].Jayasri K, CH Pooja, K Padmaja, P Eswara Prasad. Review on biochemical alterations in COVID-19 patients. International Journal of Clinical Biochemistry and Research. 2020; 7(3):307-311.

[29].Li J-W, Han T-W, Woodward M, Anderson CS, Zhou H, Chen Y-D, Neal B. The impact of 2019 novel coronavirus on heart injury: a systematic review and meta-analysis. Prog Cardiovasc Dis 2020; 63:518-524.

[30].Licata Anna, Maria Giovanna Minissale, Marco Distefano and Giuseppe Montalto. Liver injury, SARS-COV-2 infection and COVID-19: What physicians should really know? GastroHep. 2021; 3:121-130.

[31].Lippi G, Plebani M, Henry BM. Thrombocytopenia is associated with severe coronavirus disease 2019 (COVID-19) infections: A metaanalysis. Clin Chim Acta. 2020; 506:145-8.

[32].Liu Q, Wang RS, Qu GQ, et al. Gross observation report on autopsy of dead corpse system of covid-19. J Forensic Med. 2020; 36(1):21-3

[33].Ma Chunxiang, Cong Yingzi and Zhang Hu. COVID-19 and the Digestive System. Am J Gastroenterol. 2020; 115:1003-1006.

[34].Mannan Abdul Baig. Computing the effects of SARS-CoV-2 on Respiratory Regulatory Mechanisms in COVID-19. ACS Chem. Neurosci. 2020; 11:2416-2421.

[35].Mannan Abdul Baig, Areeba Khaleeq, Usman Ali, and Hira Syeda. Evidence of the COVID-19 Virus Targeting the CNS: Tissue Distribution, Host-Virus Interaction, and Proposed Neurotropic Mechanisms. ACS Chem. Neurosci. 2020; 11: 995-998.

[36]. Marcello $\mathrm{C}$ and Luisa M.Biochemical biomarkers alterations in Coronavirus Disease 2019 (COVID-19). Diagnosis. 2020; 7(4):365-372.

[37].Mirmohammadi S., A. Kianmehr, M. Arefi and A. Mahrooz. Biochemical parameters and pathogenesis of SARS-CoV-2 infection in vital organs: COVID-19 outbreak in Iran. New Microbes and New Infections. 2020; 38(C): 1-9. 


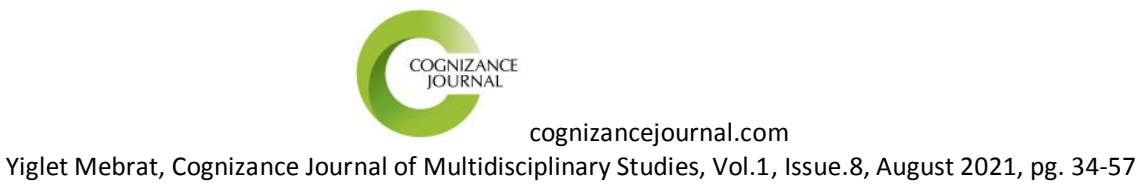

[38].Munhoz Renato Puppi, José Luiz Pedroso, Fábio Augusto Nascimento, Sergio Monteiro de almeida, Orlando Graziani Povoas Barsottini, Francisco Eduardo C Cardoso, Hélio A Ghizoni Teive. Neurological complications in patients with SARS-CoV-2 infection: a systematic review. Arq Neuropsiquiatr. 2020; 78(5):290-300.

[39].Muñoz-Durango N, Fuentes CA, Castillo AE, González-Gómez LM, Vecchiola A, Fardella CE, et al. Role of the renin-angiotensinaldosterone system beyond blood pressure regulation: molecular and cellular mechanisms involved in end-organ damage during arterial hypertension. Int J Mol Sci. 2016; 17(7):797.

[40]. Osterbur K, Mann FA, Kuroki K, DeClue A. Multiple organ dysfunction syndrome in humans and animals. J Vet Intern Med 2014; 28 (4):1141-51.

[41]. Oussalah Abderrahim, Stanislas Gleye, Isabelle Clerc Urmes, Elodie Laugel, Francoise Barbe, Sophie Orlowski, Catherine Malaplate, Isabelle Aimone-Gastin, Beatrice Maatem Caillierez, Marc Merten, Elise Jeannesson, Raphael Kormann, Jean-Luc Olivier, RosaMaria Rodriguez-Gueant, Fares Namour, Sybille Bevilacqua, Nathalie Thilly, MarieReine Losser, Antoine Kimmoun, Luc Frimat, Bruno Levy, Sebastien Gibot, Evelyne Schvoerer, Jean-Louis Gueant. The spectrum of biochemical alterations associated with organ dysfunction and inflammatory status and their association with disease outcomes in severe COVID-19: A longitudinal cohort and time-series design study. EClinicalMedicine 27. 2020; 100554:1-15.

[42].Pan X-W, Xu D, Zhang H, Zhou W, Wang L-H, Cui X-G. Identification of a potential mechanism of acute kidney injury during the COVID-19 outbreak: a study based on single-cell transcriptome analysis. Intensive Care Med. 2020; 46:1114-6.

[43].Patel KP, Patel PA, Vunnam RR, Hewlett AT, Jain R, Jing R, Vunnam SR. Gastrointestinal, hepatobiliary, and pancreatic manifestations of COVID-19. J Clin Virol. 2020; 128:1-6.

[44].Prins GH, Olinga P. Potential implications of COVID-10 in non-alcoholic fatty liver disease. Liver Int. 2020; 40:2568-2568.

[45].Post A, Dullaart RPF, Bakker SJL. Is low sodium intake a risk factor for severe and fatal COVID-19 infection? Eur J Intern Med. 2020; 75:109.

[46]. Ramos-Casals Manuel, Pilar Brito-Zerón and Xavier Mariette. Systemic and organspecific immune-related manifestations of COVID-19. Nature reviews .Rheumatology. $2021 ; 17: 315-332$.

[47].Ridruejo E, Soza A. The liver in times of COVID-19: what hepatologists should know? Ann Hepatol. 2020; 19:353-8.

[48].Sarvazad H., S. H. Cahngaripour, N. Eskandari Roozbahani and B. Izadi. Evaluation of electrolyte status of sodium, potassium and magnesium, and fasting blood sugar at the initial admission of individuals with COVID-19 without underlying disease in Golestan Hospital, Kermanshah. New Microbes and New Infections. 2020; 38(C): 1-6.

[49].Sever MY, Uzun N, Ceritli S, Mutlu H, Bayramo_glu A. Hyponatremia in COVID-19 patient using angiotensin type 1 receptor (AT1R) blocker and diuretic: a case report. J Res Clin Med 2020; 8(22):1-12.

[50].Siddiqi HK, Mehra MR. COVID-19 illness in native and immunosuppressed states: a clinical-therapeutic staging proposal. J Heart Lung Transplant. 2020; 39(5): 405-407. 


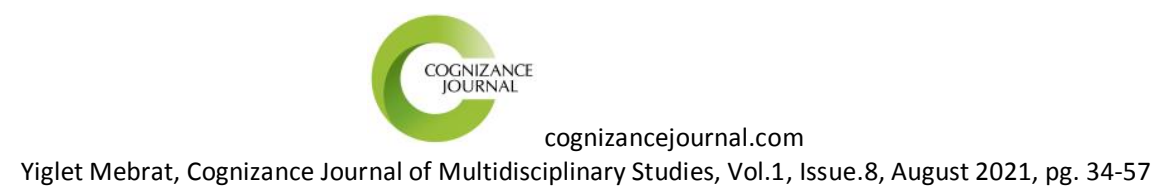

ISSN: 0976-7797

[51].Sinha, P., Matthay, M. A. \& Calfee, C. S. Is a 'Cytokine Storm' relevant to COVID-19? JAMA Intern. Med. 2020; 180: 1152-1154.

[52].Su H, Yang M, Wan C, Yi L-X, Tang F, Zhu H-Y, et al. Renal histopathological analysis of 26 postmortem findings of patients with COVID-19 in China. Kidney Int. 2020; 98:219-27.

[53].Sugimoto J, Romani AM, Valentin-Torres AM, Luciano AA, Kitchen CMR, Funderburg N, Mesiano S, and Bernstein HB.. Magnesium decreases inflammatory cytokine production: a novel innate immunomodulatory mechanism. J Immunol 2012; 188:633846.

[54].Tan L, Wang Q, Zhang D, Ding J, Huang Q, Tang YQ, Qiongshu Wang and Hongming Miao. Lymphopenia predicts disease severity of COVID-19: a descriptive and predictive study. Signal Transduct Target Ther. 2020; 5(1):1-3.

[55].Troisi Jacopo, Giorgia Venutolo, Meritxell Pujolassos Tanyà, Matteo Delli Carri, Annamaria Landolfi, Alessio Fasano. COVID-19 and the gastrointestinal tract: Source of infection or merely a target of the inflammatory process following SARS-CoV-2 infection? World J Gastroenterol. 2021; 27(14): 1406-1418.

[56]. Varga Z, Flammer AJ, Steiger P, Haberecker M, Andermatt R, Zinkernagel AS, Mehra MR, Schuepbach RA, Ruschitzka F, Moch H. Endothelial cell infection and endotheliitis in COVID-19. Lancet. 2020; 395:1417-8.

[57]. Verity Robert, Lucy C Okell, Ilaria Dorigatti, Peter Winskill, Charles Whittaker, Natsuko Imai, Gina Cuomo-Dannenburg, Hayley Thompson, Patrick G T Walker, Han Fu, Amy Dighe, Jamie T Griffin, Marc Baguelin, Sangeeta Bhatia, Adhiratha Boonyasiri, Anne Cori, Zulma Cucunubá, Rich FitzJohn, Katy Gaythorpe, Will Green, Arran Hamlet, Wes Hinsley, Daniel Laydon, Gemma Nedjati-Gilani, Steven Riley, Sabine van Elsland, Erik Volz, Haowei Wang, Yuanrong Wang, Xiaoyue Xi, Christl A Donnelly, Azra C Ghani, Neil M Ferguson. Estimates of the severity of coronavirus disease 2019: a model-based analysis. Lancet Infect Dis 2020; 20: 669-77.

[58]. Villapol S. Gastrointestinal symptoms associated with COVID-19: impact on the gut microbiome. Transl Res. 2020; 226: 57-69

[59]. Wang D, Hu B, Hu C, Zhu F, Liu X, Zhang J, Wang B, Xiang H, Cheng Z, Xiong Y, Zhao Y, Li Y, Wang X, Peng Z. Clinical Characteristics of 138 Hospitalized Patients With 2019 Novel CoronavirusInfected Pneumonia in Wuhan, China. JAMA. 2020; 323: 1061-1069.

[60].Wei Feng, Ashley M. Newbigging, Connie Le, Bo Pang, Hanyong Peng, Yiren Cao, Jinjun Wu, Ghulam Abbas, Jin Song, Dian-Bing Wang, Mengmeng Cui, Jeffrey Tao, D. Lorne Tyrrell, Xian-En Zhang, Hongquan Zhang, and X. Chris Le. Molecular Diagnosis of COVID-19: Challenges and Research Needs. Anal. Chem. 2020; 92: 10196-10209.

[61].Wiersinga, W. J., Rhodes, A., Cheng, A. C., Peacock, S. J. \& Prescott, H. C. Pathophysiology, transmission, diagnosis, and treatment of coronavirus disease 2019 (COVID-19): a review. JAMA. 2020; 324: 782-793.

[62].Wong Yu Jun, Malcolm Tan, Qishi Zheng, James Weiquan Li, Rahul Kumar, Kwong Ming Fock, Eng Kiong Teo, Tiing Leong Ang. A systematic review and meta-analysis of the COVID-19 associatedliver injury. Annals of Hepatology. 2020; 19: 627-634. 


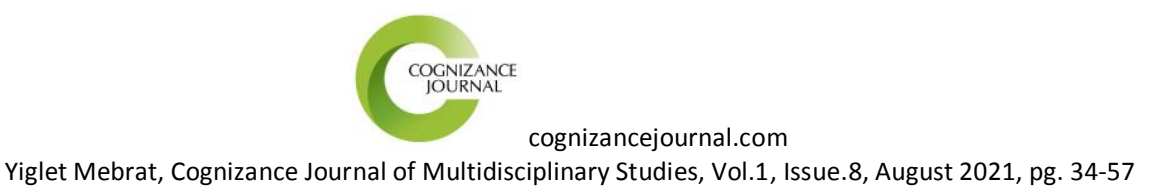

[63]. Wu P, Duan F, Luo C, Liu Q, Qu X, Liang L, Wu K. Characteristics of ocular findings of patients with coronavirus disease 2019 (COVID-19) in Hubei Province, China. JAMA Ophthalmol. 2020; 138(5): 575-578.

[64]. Yang H, Yang LC, Zhang RT, Ling YP, Ge QG. Risks factors for death among COVID19 patients combined with hypertension, coronary heart disease or diabetes. Beijing Da Xue Xue Bao 2020; 52:420-4.

[65].Zeyed YF, Bastarache JA, Matthay MA, Ware LB. The severity of shock is associated with impaired rates of net alveolar fluid clearance in clinical acute lung injury. Am $J$ Physiol Lung Cell Mol Physiol. 2012; 303:L550-5.

[66].Xu L, Liu J, Lu M, Yang D, Zheng X. Liver injury during highly pathogenic human coronavirus infections. Liver Int. 2020; 40:998-1004.

[67].Zhang C, Shi L, Wang FS. Liver injury in COVID-19: management and challenges. Lancet Gastroenterol Hepatol. 2020; 5:428-30. 